\title{
Thread based microfluidic platform for urinary creatinine analysis
}

\author{
Manuel J. Arroyo ${ }^{\mathrm{a}}$, Miguel M. Erenas ${ }^{\mathrm{a}, \mathrm{b}, *}$, Ignacio de Orbe-Payáa, ${ }^{a, b}$, Kevin \\ Cantrell $^{\mathrm{c}}$, Jose A. Dobado ${ }^{\mathrm{d}}$, Pablo Ballester ${ }^{\mathrm{e}, \mathrm{f}}$, Pascal Blondeau ${ }^{\mathrm{g}}$, Alfonso \\ Salinas-Castillo ${ }^{a, b}$, Luis Fermín Capitán-Vallvey ${ }^{\mathrm{a}, \mathrm{b}}$ \\ ${ }^{a}$ ECsens, Department of Analytical Chemistry, Campus Fuentenueva, Faculty of Sciences, \\ 18071 University of Granada, Granada, Spain. \\ ${ }^{b}$ Unit of Excellence in Chemistry applied to Biomedicine and the Environment of the \\ University of Granada. \\ ${ }^{c}$ Department of Chemistry. The University of Portland, $5000 \mathrm{~N}$ Willamette Blvd, Portland, \\ OR 97203 USA. \\ ${ }^{d}$ Grupo de Modelización y Diseño Molecular. Department of Organic Chemistry, Faculty of \\ Sciences, University of Granada, 18071 Granada, Spain. \\ ${ }^{e}$ Institute of Chemical Research of Catalonia (ICIQ). The Barcelona Institute of Science \\ and Technology. Av. Països Catalans, 16, 43007 Tarragona, Spain. \\ ${ }^{f}$ Catalan Institution for Research and Advanced Studies (ICREA) Pg. Lluís Companys 23, \\ 08010 Barcelona, Spain. \\ ${ }^{g}$ Departament de Química Analítica i Química Orgànica, Universitat Rovira $i$ Virgili \\ (URV), C/ Marcel·lí Domingo 1, 43007 Tarragona, Spain.
}

\begin{abstract}
Creatinine level in urine is a key factor to monitor kidney performance. The use of an alternative microfluidic platform based on cellulose substrates is an interesting option to integrate sample treatment, creatinine recognition by ionophore extraction chemistry and quantification by color measurement through consumer electronics imaging devices. The inclusion of ionophore extraction chemistry based on aryl-substituted calix[4]pyrrole synthetic receptor on $8.7 \mathrm{~mm}$ long cotton thread permit the sample treatment, optical recognition of creatinine and their quantification by smartphone running app in unfiltered urine samples diluted 1:100 ratio. The device shows a short response time, $30 \mathrm{~s}$, to creatinine over a wide dynamic range (from $1.6 \times 10^{-6}$ to $5 \times 10^{-2} \mathrm{M}$ ) with reproducibility between $2.9-4.3 \%$. The low interference level of representative species in urine is studied and justified by density functional theory (DFT) calculations.
\end{abstract}

\footnotetext{
${ }^{*}$ Corresponding author

Email address: erenas@ugr.es (Miguel M. Erenas)
} 
Keywords: Optical device, $\mu \mathrm{TAD}$, creatinine determination, Density Functional Theory, calix[4]pyrrole derivative, smartphone

\section{Introduction}

Creatinine is a key indicator of renal function and its quantification based on enzymes or chromogenic reactions has various drawbacks, [1 justifying the search for new detection schemes to simplify the procedure without compromis5 ing the analytical characteristics. The design of creatininium cation receptors has been described in the literature. 2] These receptors are based on a nitrogen heterocycle with one reversible binding site that produces a colour change upon a proton transfer.

However, another family of receptors based on calix[4]pyrrole has been extensively used and reported, 3] yielding two binding sites at the receptor (the upper and lower rim of the receptor, see Figure 11). Ionophores have been widely used for electrochemical and optical ion recognition for the determination of inorganic cations and anions and, to a lesser extent, organic ions. In all cases, the optical transduction is performed by means of a lipophilic $\mathrm{pH}$ indicator due to

15 an ion exchange or co-extraction equilibrium initiated by an electroneutrality condition in the hydrophobic sensing membrane. 4, 5]

The need to move the analyte from the solution to the bulk of the membrane produces one of the drawbacks of these sensors, their long response time. To reduce the response time, different strategies have been devised, typically based on increasing the contact surface and reducing the molecular diffusion distance. 6

One interesting option to achieve a short response time as well as an efficient interaction is the inclusion of ionophore-based chemistry in a microfluidic analytical device, which miniaturizes the laboratory process aimed at detecting

25 a specific target by converting a recognition event into a detectable quantitative signal. Some examples have appeared in the literature about the use of microfluidic platforms combined with ionophore-based optical sensors: a) a centrifugal 
platform for $\mathrm{K}^{+}$determination; [7, 8, b) a pressure-driven platform for alkaline ions, 9] $\mathrm{Cd}^{2+}$, or $\mathrm{Hg}^{2+}$; 10 c) a segmented flow microfluidic platform for $\mathrm{K}^{+}$, so $\mathrm{Na}^{+}, \mathrm{Cl}^{-}$and protamine; [1] and d) a capillary microfluidic platform for $\mathrm{K}^{+}$. [6, 12 .

However, not all microfluidic platforms based on ionophore extraction recognition are suitable for reducing the response time. The droplet microfluidicsbased sensing scheme reduces the reaction time from $10 \mathrm{~min}$ to about $1 \mathrm{~s}$. [11]

35 Other examples include the pressure-driven laminar flow platform for alkaline ions with $8 \mathrm{~s}$ response time [9] and the thread-based capillary platform for $\mathrm{K}^{+}$, with requires $15 \mathrm{~s}$ instead of the usual $5 \mathrm{~min}$ in membrane format. [6]

This paper describes the use of an ionophore for creatinine in a microfluidic analytical device. The ionophore is an aryl-substituted monophosphonate40 bridged calix[4]pyrrole (CalixPyr), previously reported by some of us, 3] and applied in an ion-selective potentiometric sensor, [13] as well as in an optical disposable sensor to monitor creatinine levels in urine. [14]

The inclusion of ionophore-based chemistry in a thread-based microfluidic analytical device ( $\mu \mathrm{TAD})$ in single-use format is an interesting option whenever 45 an efficient interaction, easy sample treatment and a short response time is desired. The recognition strategy used is combined with colour measurement with a consumer electronics imaging device, such as a digital camera or a smartphone, which makes it possible to use chromatic coordinates as an analytical parameter and avoids the need to work with normalized signals, which considerably 50 simplifies the use of the device. [6]

On the other hand, the lack of experimental information with respect to the binding mechanism of these compounds (ionophore $\cdots$ creatininium ion) requires the use of computational chemistry tools to provide further insight into the structure and formation of different complexes on both sides of the ionophore, 55 as well as the role of the interference with other analytes such as urea, uric acid and simple cations $\left(\mathrm{Na}^{+}, \mathrm{K}^{+}, \mathrm{NH}_{4}^{+}\right.$) (see Figure 1). The formation of trimers at the two binding sites of the ionophore merits special attention, as it provides the most basic information about the binding mechanism. Density 
functional theory (DFT) calculations have been reported to accurately describe
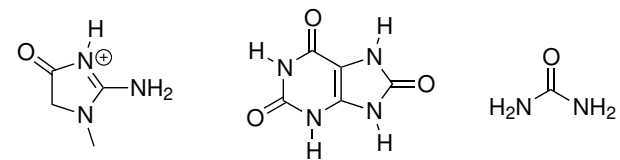

Creatininium cation

Uric acid

Urea

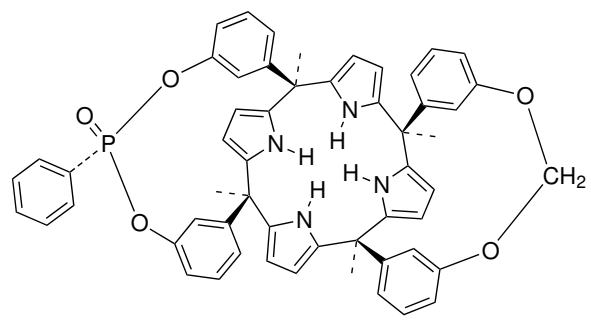

Calix[4]pyrrole derivative

Figure 1: Scheme of the protonated creatinine molecule (creatininium cation), uric acid, urea and aryl-substituted monophosphonate-bridged calix[4]pyrrole derivative (CalixPyr) studied.

With respect to the tautomeric equilibrium of creatinine and creatininium cation, DFT calculations have been explored, 17, 18, reporting that the preferred tautomeric form of the creatininium cation (creat) consists of the monoprotonated form with an $-\mathrm{NH}$ group (see Figure 1).

\section{Experimental}

\subsection{Reagents and materials}

All reagents used for cocktail and solutions preparation were analytical grade and purchased from Sigma-Aldrich (Madrid, Spain). Poly(vinylchloride) (PVC; high molecular weight), 4',5'-dibromofluorescein octadecyl ester (ETH7075), potassium tetrakis-[3,5-bis-(tri-fluoro-methyl)-phenyl]-borate (KFMPB), o-nitrophenyl-octyl-ether (NPOE), tetrahydrofuran (THF) and KTFPB, together with the ionophore CalixPyr for creatinine, were synthesized in our labs according to Guinovart et al. 33. A fixed volume of the sensing cocktail was cast on the Mylar type support (Goodfellow, Cambridge, UK) as well as white cotton Finca 
thread (Presencia Hilaturas S.A. Alzira, Valencia, Spain) used as support, made up of $250 \pm 10$ fibres and around $600 \mu \mathrm{m}$ thick.

All the aqueous solutions were prepared in purified water $(18.2 \mathrm{M} \Omega \cdot \mathrm{cm}$ resistance) obtained from a Milli-RO 12 plus Milli-Q station (Millipore, Bedford, MA, USA). All the interference solutions were prepared by $\mathrm{K}^{+}, \mathrm{Na}^{+}$and $\mathrm{NH}_{4}^{+}$

so as chlorides, uric acid and urea in water. $\mathrm{pH} 3.8$ acetic acid/sodium acetate buffer $0.1 \mathrm{M}$ was prepared diluting acetic acid in water and the $\mathrm{pH}$ was adjusted by adding $\mathrm{NaOH} 0.1 \mathrm{M}$. Sodium carbonate $10 \mathrm{mg} \cdot \mathrm{mL}^{-1}$ solution was also used to scour the cotton thread.

\subsection{Instruments and software}

The sensing membranes were prepared by spin-coating using WS-400Bz6NNP/LITE equipment (Laurell Technologies Corporation, PA, USA). Digitalization of the sensing membranes and $\mu \mathrm{TAD}$ was done using a Sony DSC-HX300 (Sony, Tokyo, Japan) digital camera and a Samsung Galaxy S5 smartphone (Samsung Electronics, Suwon, South Korea), and picture files were obtained and 90 analysed using ImageJ (National Institutes of Health) software. To perform the statistical and mathematical treatment, OriginPro v.8 (OriginLab Corporation, USA) software and an Microsoft Excel spreadsheet were used. A Rayjet Trotec Laser engraving printer (Trotec, Austria), commanded by Rayjet Commander software, was used to design and fabricate the holder for the $\mu \mathrm{TAD}$.

FEG-ESEM, QuenScan 650F FEI ${ }^{\circledR}$ electronic microscope together with an Everhart-Thornley detector (ETD), circular backscatter detector (CBS) and energy-dispersive detector (EDS) were used to obtain images from the thread with and without the sensing cocktail to discover the size, morphology and distribution along the thread. All of these studies were done at the Centre for Scientific Instrumentation (University of Granada, Spain).

\section{3. $\mu T A D$ preparation}

The sensing cocktail was prepared by dissolution of $0.88 \mathrm{mg}(0.9 \mathrm{wt}$. \%) of ETH7075, $3.2 \mathrm{mg}$ (3.2 wt. \%) of CalixPyr, $1.5 \mathrm{mg}$ (1.5 wt. \%) of KFMPB, 63.9 
mg (63.0 wt. \%) of NPOE, and $31.9 \mathrm{mg}$ (31.5 wt. \%) of KTFPB, in $1 \mathrm{~mL}$ of freshly distilled THF. 14, 3. The cotton thread used as the support was first scoured by boiling in an aqueous solution of $10 \mathrm{mg} \cdot \mathrm{mL}^{-1}$ of $\mathrm{Na}_{2} \mathrm{CO}_{3}$ for $5 \mathrm{~min}$ to remove the thread waxes. The thread was then washed several times until the rinsate was $\mathrm{pH}$ neutral. Finally, it was sonicated 3 times in purified water for $5 \mathrm{~min}$ and left to dry at room temperature. To prepare the $\mu \mathrm{TAD}, 5 \mu \mathrm{L}$ of $\mathrm{pH} 3.8$ acetic acid/ sodium acetate buffer $(0.1 \mathrm{M})$ were added to a $25-\mathrm{mm}$ long thread and after drying, $0.5 \mu \mathrm{L}$ of the sensing cocktail was added in the same place and left to dry. The thread was then placed in a holder designed and made by us for easier use (see Figure 2). This was done by engraving a piece of black methacrylate $(20 \times 20 \times 4 \mathrm{~mm})$ using a laser engraving printer.

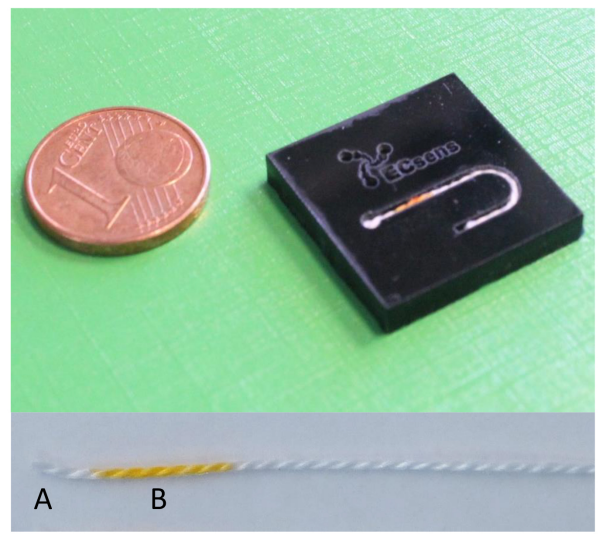

Figure 2: Picture of the $\mu \mathrm{TAD}$ designed for the quantitative determination of creatinine in urine samples. A) sampling zone, B) sensing zone.

\subsection{Measurement setup}

To optimize the assay conditions, $\mu \mathrm{TAD}$ was used by adding $10 \mu \mathrm{L}$ of the sample or standard dropped on one end of the thread and then digitalized after $30 \mathrm{~s}$ using the Sony DSC-HX300 camera placed in a custom cubic light box illuminated by two LED light bulbs $(3000 \mathrm{~K})$ in a fixed position. The camera was set up as follows: resolution of $3648 \times 2736$ pixels, aperture value $\mathrm{f} / 3.5$, exposure 
time $1 / 40 \mathrm{~s}$, ISO-80, $2800 \mathrm{~K}$ white balance (see Section S1), with the images saved in jpg format (Joint Photographic Experts Group). The image obtained was analysed using Image J software to select the region of interest (ROI) of the $\mu \mathrm{TAD}$ and analyse it. The proposed procedure for creatinine determination is based on the use of a Samsung Galaxy S5 smartphone, (Samsung Electronics, Suwon, South Korea) running an in-house app that automatically detects the ROI of the $\mu \mathrm{TAD}$ and perform the colorimetric measurement. [19] Once the picture is obtained, it is transformed to the HSV colour space from the RGB colour space, using the hue channel used to identify the ROI. The pixels inside this ROI are used to calculate the analytical parameter.

\subsection{Analysis of real samples}

Freshly obtained unfiltered urine samples from healthy volunteers were analysed after prior dilution at 1:100 ratio in water, obtaining three replicates each, using three different $\mu \mathrm{TAD}$. The results obtained were compared with the predicted ones, using Jaffé's method as the reference. In the case of Jaffé, the samples where diluted and filtered before use. 20]

All experiments were performed in compliance with the relevant laws and institutional guidelines, being no necessary the specific approval of a committee due to the very small scale routine test. Urine samples were obtained with informed consent of the volunteers.

\section{Computational details}

Density functional theory (DFT) calculations were performed using the ORCA 4.0.1 [21, 22] computational program. All the geometry optimizations of complexes 1-18 and their electronic properties were performed using the ORCA 145 4.0.1 [21, 22] program. PBEh-3c [23] functional was employed together with the def2-mSVP basis function to compute optimized geometries, energies and frequency calculations. The PBEh-3c method includes correction of the basis set superposition error (BSSE) 24] with the gCP [25] algorithm, as well as atompairwise dispersion correction with the Becke-Johnson damping scheme (D3BJ). 
[26, 27. Stationary points were characterized by calculating the eigenvalues of the second-derivative matrix, i.e., Hessian. None of the energy minima showed any imaginary frequency. Binding energies $\left(E_{b i n d}\right)$ indicate the intensity of the interaction between CalixPyr moiety and different analytes: creatininium ion, $\mathrm{Na}^{+}, \mathrm{K}^{+}$and $\mathrm{NH}_{4}{ }^{+}$cations, and urea and uric acid molecules. $E_{\text {bind }}$ were calculated according to the Equation 1 :

$$
E_{\text {bind }}=E_{\text {complex }}-\left(E_{\text {CalixPyr }}+E_{\text {analyte }}\right)
$$

where $E_{\text {bind }}, E_{\text {complex }}, E_{\text {CalixPyr }}$ and $E_{\text {analyte }}$ represent the binding energy, the total energy of the system (CalixPyr- - - -analyte complex) and the energies of the isolated CalixPyr and cation moieties, respectively.

\section{Results and discussion}

The developed $\mu \mathrm{TAD}$ is based on ionophore-chromoionophore chemistry, where the creatininium ion interacts selectively with the CalixPyr ionophore inside the membrane. To hold the electroneutrality condition in the membrane, $\mathrm{a}^{+}$from the lipophilic $\mathrm{pH}$ indicator is lost, turning the colour from yellow to orange. 14]

$$
\overline{p L}+\overline{H_{2} I^{+}}+\overline{R^{-}}+H C^{+} \longleftrightarrow \overline{H I}+\overline{H C L_{p}^{+}}+\overline{R^{-}}+H^{+}
$$

Equation 2 shows the recognition process where the species present in the membrane are barred and $L$ is the ionophore, $\mathrm{H}_{2} \mathrm{I}^{+}$is the lipophilic $\mathrm{pH}$ indicator and $\mathrm{R}^{-}$the lipophilic anion, and the reaction with creatininium ions $\left(\mathrm{HC}^{+}\right)$in solution proceeds to form the species $\mathrm{HCL}_{\mathrm{p}}^{+}$.

DFT calculations were carried out to characterize the structure and binding energies $\left(E_{b i n d}\right)$ of a series of complexes between CalixPyr as a host molecule and different guests: creatininium cation $(\mathbf{1})$, uric acid (2), urea $(\mathbf{3}), \mathrm{Na}^{+}(\mathbf{4}), \mathrm{K}^{+}$ (5) and $\mathrm{NH}_{4}^{+}(\mathbf{6})$ (see Figure 3). For the complexes studied between CalixPyr and uric acid: $(\mathbf{2}, \mathbf{8}, \mathbf{1 1}, \mathbf{1 2}$ and 14), two orientations of the uric acid moiety with respect to the CalixPyr were considered (named $\mathbf{a}$ and $\mathbf{b}$, see Section 


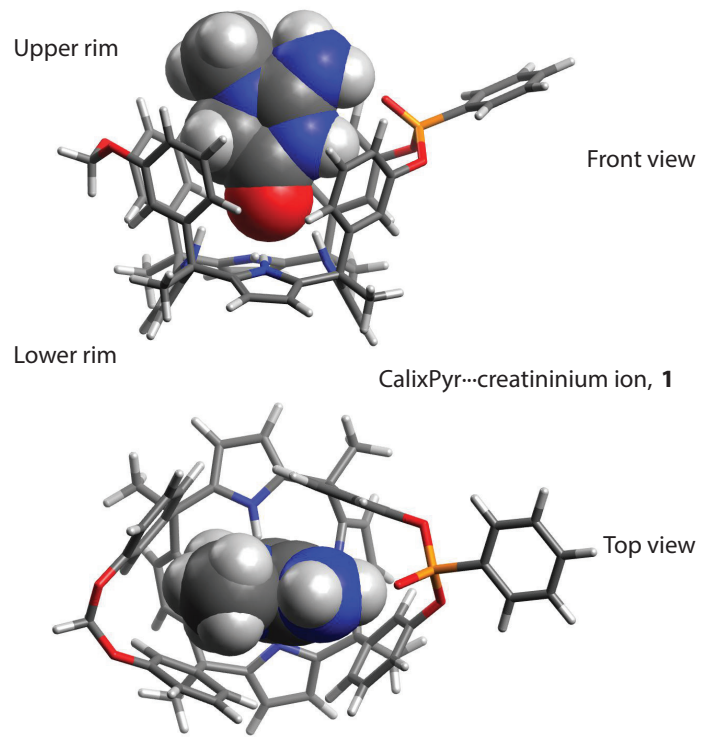

Figure 3: Top and front views of the structure of [CalixPyr $\cdots$ creat $]^{+}(\mathbf{1})$ complex, optimized at the DFT theoretical level (PBEh-3c).

S10). Table 1 summarizes the $E_{\text {bind }}$ DFT values, showing larger values for complexes 1-3, at the upper rim of CalixPyr, compared to the complexes formed at the lower rim. However, the results obtained for simple cations such as $\mathrm{Na}^{+}$, $\mathrm{K}^{+}$and $\mathrm{NH}_{4}^{+}$showed larger $E_{\text {bind }}$ values at the lower rim of the CalixPyr moiety, also yielding the largest $E_{\text {bind }}$ values: $-75.9,-62.5$ and $-61.0 \mathrm{kcal} \cdot \mathrm{mol}^{-1}$, for complexes 4, 5 and $\mathbf{6}$, respectively. The complex CalixPyr $\cdots$ creatininium cation (1) showed a preference for the upper rim binding site with an $E_{b i n d}$ value of $-56.7 \mathrm{kcal} \cdot \mathrm{mol}^{-1}$. Charged species resulted in larger $E_{\text {bind }}$ values. However, complex $\mathbf{2}$ at the upper rim showed closer values compared with $\mathbf{5}$, indicating than the charge is not the only driving force at the host-guest interaction.

The 1- $\alpha$ parameter is typically used as the analytical parameter to be related to the activity of the analyte (see Equation 3). This is defined as the ratio of protonated chromoionophore to its analytical concentration, measured by an 
Table 1: Binding energies $\left(E_{b i n d}\right)$ for the different binary CalixPyr rims of the complexes $(\mathbf{1}-$

6) [CalixPyr $\cdots$ analyte] (creatininium cation, uric acid, urea, $\mathrm{Na}^{+}, \mathrm{K}^{+}$and $\mathrm{NH}_{4}^{+}$), calculated at the DFT theoretical level (PBEh-3c)

\begin{tabular}{lcc}
\hline System & \multicolumn{2}{c}{$E_{\text {bind }}\left(\mathrm{kcal} \cdot \mathrm{mol}^{-1}\right)$} \\
\cline { 2 - 3 } & upper rim & lower rim \\
\hline $\mathbf{1},[\text { CalixPyr } \cdots \text { creat }]^{+}$ & -56.7 & -34.3 \\
$\mathbf{2 a},[$ CalixPyr $\cdots$ uric ac. $]$ & -50.8 & -16.2 \\
$\mathbf{2 b},[$ CalixPyr $\cdots$ uric ac.] & -42.5 & -17.2 \\
$\mathbf{3},[$ CalixPyr $\cdots$ urea] & -46.5 & -14.3 \\
$\mathbf{4},[\text { CalixPyr } \cdots \mathrm{Na}]^{+}$ & -59.9 & -75.9 \\
$\mathbf{5},\left[\right.$ CalixPyr $\cdots \mathrm{K}^{+}$ & -53.7 & -62.5 \\
$\mathbf{6},\left[\text { CalixPyr } \cdots \mathrm{NH}_{4}\right]^{+}$ & -55.8 & -61.0 \\
\hline
\end{tabular}

optical property such as absorption, reflection or emission. 28,

$$
1-\alpha=\frac{X-X_{H I}}{X_{H_{2} I^{+}}-X_{H I}}
$$

This normalized signal involves measuring the optical signals related to fully protonated $\left(\mathrm{X}_{\mathrm{H} 2 \mathrm{I}+}\right)$ and deprotonated $\left(\mathrm{X}_{\mathrm{HI}}\right)$ species in addition to the signals coming from the sample or standards $(\mathrm{X})$.

When using this recognition chemistry in single-use devices or disposable sensors, working with normalized signals is a cumbersome or even impossible task, because they require the acquisition of different measurements with the same device. To solve this problem, we propose using the chromatic coordinate $H$ from the hue-oriented $H S V$ colour space as the analytical parameter, 6] carrying out the analyte measurements with no need to activate the membrane or normalize the signal. [29, 30] To use the tonal coordinate $H$ as the analytical parameter, a tone change must occur due to deprotonate the indicator, not a simple change of intensity. In this case, the lipophilic indicator selected, ETH7075, 31, 32 has a tonal colour change from yellow to orange when it loses a proton.

To confirm the possible use of the parameter $H$ in this sensing system, the 
values of parameter $1-\alpha$ and $H$, of activated and non-activated membranes prepared on Mylar, were compared at different creatinine concentrations (see Section S2). Figure 4 A shows the evolution of $1-\alpha$ calculated both from absorbance measurements using a spectrophotometer and from the $H$ coordinate obtained digitalizing the membrane with a camera. Figure $4 \mathrm{~B}$ shows the evolution of $\mathrm{H}$ prior to activation of the membrane in $\mathrm{HCl}$ and without any activation. Although the values were different, the shape of the creatinine dependence concentration was the same, confirming that $H$ can be used directly as an analytical parameter without any type of activation of the membrane, as described in the literature. 29]

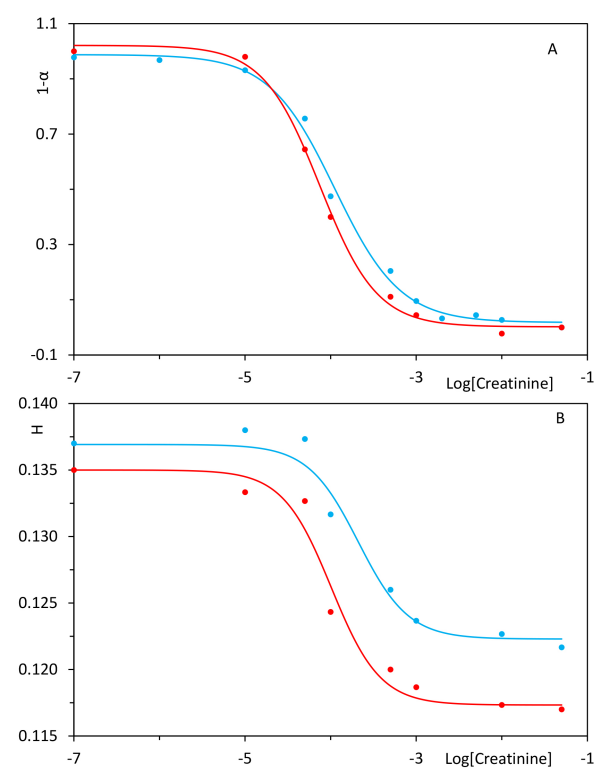

Figure 4: Calibration obtained from activated membrane (blue line) and without activation (red line), using A) 1- $\alpha$, and B) $H$ as analytical parameters.

One of the objectives of this study is to design a microfluidic device for cre215 atinine determination using cotton thread as a cheap and eco-friendly support. Thread-based microfluidic devices have clear advantages in terms of the reaction time and volume of the sample and reagents needed, as well as the possibility 
of including different analytical operations, such as sample buffering, analyte preconcentration [33, 34, 35] and separation. [19]

To prepare the $\mu \mathrm{TAD}, 0.5 \mu \mathrm{L}$ of cocktail is sufficient to create a sensing area with good reproducibility in length $(8.7 \pm 0.6 \mathrm{~mm} ; \mathrm{n}=55)$ and number of pixels when photographed (average ROI $6437 \pm 115$; $\mathrm{n}=55$ ). The composition of the cocktail for creatinine determination was previously optimized [14] and once added to and dried on the thread, the PVC membrane contains $22537.1 \mathrm{mmol} \cdot \mathrm{kg}^{-1}$ ionophore, $12.4 \mathrm{mmol} \cdot \mathrm{kg}^{-1}$ lipophilic $\mathrm{pH}$ indicator, and 17.4 mmol $\cdot \mathrm{kg}^{-1}$ lipophilic salt. [14 A variation in the composition of the cocktail can affect the efficiency of the $\mu \mathrm{TAD}$ in terms of selectivity pattern and variation in the flow of the fluid through the thread. To select the right amount of membrane components deposited on the thread while maintaining the optimized ionophore:chromoionophore:lipophilic salt ratio, serially diluted cocktail solutions were studied. The cocktails prepared by diluting the optimized cocktail were 1:0, 1:1, 1:2, 1:3 and 1:4 (optimized cocktail: THF), of which $0.5 \mu \mathrm{L}$ was deposited on the thread, causing it to react with $1 \times 10^{-7}, 1 \times 10^{-4}$ and $5 \times 10^{-2} \mathrm{M}$ creatinine solutions. The cocktail with the best results in terms of signal range variation of $H$ value (0.015) and CV (around 0.7\%) was the non-diluted one (see Table S1).

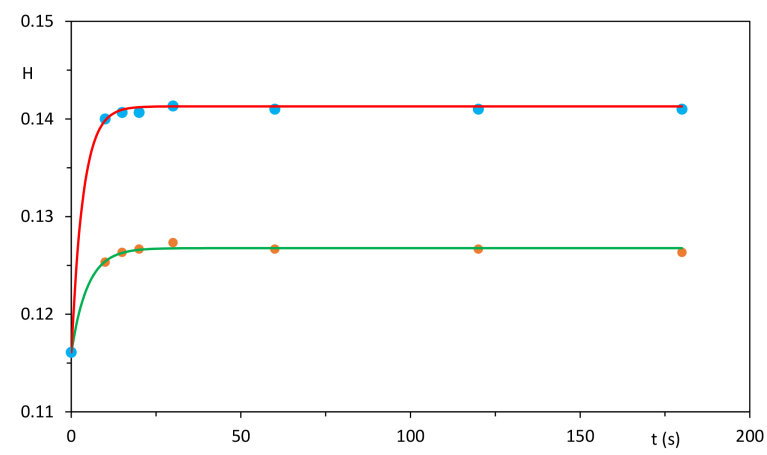

Figure 5: Equilibration time at high (green line) and low creatinine (red line) concentration, and the data set obtained. 
We previously demonstrated that 3.8 is the optimum $\mathrm{pH}$ for electrochemical and optical sensing membranes based on the use of the CalixPyr ionophore. [13, 14] Using thread makes it possible to include $\mathrm{pH}$ adjustment as an analytical EDX spectrum 6] (see Section S5). The increase in the contact surface and the small size of the sensing membrane (around $1.2 \mu \mathrm{m}$ thick) explains the device's rapid ion exchange and short response time. 
The $H$ signal variation in this device is small (0.015), but it is possible to obtain it with good precision, which is why the $H$ value obtained after $30 \mathrm{~s}$ of the $\mu$ TAD equilibration with creatinine was used as the analytical parameter. To characterize the sensing device, a calibration was carried out with 11 creatinine standard solutions with 5 replicates each (see Section S6). Figure6 presents the experimental data obtained as well as its fit to a sigmoidal Boltzmann equation (see eq. 4 and Table 2) with an $R^{2}$ of 0.976 .

$$
H=\frac{A_{1}-A_{2}}{1+e^{\frac{\log \mid \text { Creatinine } \mid-A_{3}}{A_{4}}}}+A_{2}
$$

Table 2: Analytical parameters and Boltzmann equation for creatinine $\mu \mathrm{TAD}$

\begin{tabular}{cr} 
Analytical parameters & \\
\hline Boltzmann equation (see eq. 4 \\
\hline$A_{1}$ & 0.1378 \\
$A_{2}$ & 0.1213 \\
$A_{3}$ & -3.135 \\
$A_{4}$ & 1.172 \\
$\mathrm{R}^{2}$ & 0.976 \\
LOD & $1.6 \times 10^{-6} \mathrm{M}$ \\
Dynamic range & $1.6 \times 10^{-6}-5 \times 10^{-2} \mathrm{M}$ \\
\hline Precision & $\mathrm{n}=5$ \\
\hline $1 \times 10^{-5} \mathrm{M}$ & $3.9 \%$ \\
$5 \times 10^{-4} \mathrm{M}$ & $4.3 \%$ \\
$1 \times 10^{-2} \mathrm{M}$ & $2.9 \%$ \\
\hline
\end{tabular}

The figures of merit (see Table 2 ) of the $\mu$ TAD were calculated obtaining a CV around $4 \%(\mathrm{n}=5)$ and a LOD of $1.6 \times 10^{-6} \mathrm{M}$. This LOD is lower than 275 the one obtained from optical sensing membranes on Mylar $\left(1.0 \times 10^{-5} \mathrm{M}\right)$ as is the precision in terms of $\mathrm{CV}$, which when using an optical sensing membrane on Mylar was around 6\%. 14] 


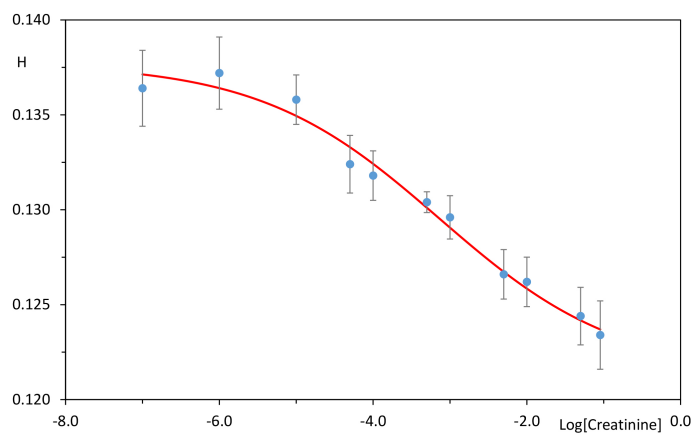

Figure 6: Calibration of the $\mu \mathrm{TAD}$ using $H$ as the analytical parameter $(\mathrm{n}=5)$.

\subsection{Interferences}

Different interfering compounds were studied to characterize the selectivity of the $\mu \mathrm{TAD}$ against representative species that can be found in urine, such as sodium, potassium, ammonium, uric acid and urea (see Section S7). The separate solutions method was used to calculate the selectivity coefficients $\left(K_{c, j}\right)$ as well as the maximum tolerable error $\left(P_{I J}\right)$ for each interfering compound compared to creatinine ( $\left.K_{\text {exch }} 0.25\right)$. The results obtained (see Table 3 ) showed that the $\mu \mathrm{TAD}$ does not respond to $\mathrm{Na}^{+}$and uric acid even at high concentration, and in the case of $\mathrm{K}^{+}$and $\mathrm{NH}_{4}{ }^{+}$the $K_{\text {exch }}$ 's were two magnitude order lower and four in the case of urea. Finally, considering that the maximum tolerable error $\left(P_{I J}\right)$ in urine is around $10 \%, 13$, the $P_{I J}$ 's were calculated for each interfering compound at 1, 10 and $100 \%$ error (see Table 3). In the case of $\mathrm{NH}_{4}{ }^{+}, \mathrm{K}^{+}$and urea, the error was lower than $10 \%$.

After studying the interference species, an artificial urine was prepared (see Table S4), studying how the presence of all the species in the same sample affects the $\mu \mathrm{TAD}$ response. Artificial urine was analysed adding $10 \mu \mathrm{L}$ to 9 different $\mu$ TADs, obtaining a creatinine concentration of $0.9 \pm 0.3 \mathrm{mM}$. The usual species present in artificial urine did not appear to affect the behaviour of the $\mu$ TAD.

The effect of the interference species can be studied using DFT calculations. For this purpose, different ternary systems were characterized. The resulting 
Table 3: $K_{\text {exch }}$ obtained for the interfering compound considered and the $\log K_{C, j}$ obtained by the separated solutions method, and the $P_{C, j}$ calculated for three different tolerable maximum error

\begin{tabular}{lccccc}
\hline \multirow{2}{*}{ Interfering } & $K_{\text {exch }}$ & $\log K_{C, j}$ & \multicolumn{3}{c}{ Required $P_{C, j}$} \\
\cline { 3 - 6 } & & & $1 \%$ & $10 \%$ & $100 \%$ \\
\hline $\mathrm{K}^{+}$ & $1.4 \times 10^{-3}$ & -2.3 & -3.3 & -2.3 & -1.3 \\
$\mathrm{Na}^{+}$ & - & - & -3.6 & -2.6 & -1.6 \\
$\mathrm{NH}_{4}^{+}$ & $2.3 \times 10^{-3}$ & -2.1 & -2.6 & -1.6 & -0.6 \\
Uric acid & - & - & -1.9 & -0.9 & 0.1 \\
Urea & $1.9 \times 10^{-5}$ & -4.1 & -3.7 & -2.7 & 1.7 \\
\hline
\end{tabular}

$E_{b i n d}$ values are summarized in Table 4. The results indicated that the creatininium ion had strong preference to bind to CalixPyr moiety at the upper rim when urea was present at the lower rim (see Figure 7), yielding $E_{b i n d}$ values of -47.9 and $-37.5 \mathrm{kcal} \cdot \mathrm{mol}^{-1}$ of complex 9 for the creatininium and urea, respectively. Also, complex 13 presented the largest $E_{b i n d}$ value for the creatininium ion $-59.5 \mathrm{kcal} \cdot \mathrm{mol}^{-1}$ at the upper rim, when urea was bound to the lower rim. From all the above, one can conclude that the $E_{\text {bind }}$ values for the creatininium ion with the CalixPyr host were enhanced when interference species such as urea were present, yielding a synergistic effect that augmented the creatininium affinity at the CalixPyr.

Finally, the $\mu \mathrm{TAD}$ was applied to real urine samples. Although the results from artificial urine shows that the $\mu \mathrm{TAD}$ should work directly in urine, the experimental results, in agreement with literature, [13, 14] show that some unidentified components interfere, resulting in a higher concentration of creatinine than expected from Jaffé method. 20 Two different strategies were used in order to avoid the interference: urine dilution and urine filtration (see Section S8). Samples were tested at different water dilutions (1:0, 1:50 and 1:100) in addition to filtered and non-filtered samples. The evolution of the signal over time was also studied to confirm that the signal is steady at $30 \mathrm{~s}$. Creatinine concentration in urine, obtained by the $\mu \mathrm{TAD}$, was compared to the results 
Table 4: Binding energies $\left(E_{\text {bind }}\right)$ for the different ternary CalixPyr complexes $(\mathbf{7}-\mathbf{1 8})$ [analyte... CalixPyr $\cdots$ analyte] (creatininium cation, uric acid, urea, $\mathrm{Na}^{+}, \mathrm{K}^{+}$and $\mathrm{NH}_{4}^{+}$), calculated at the DFT theoretical level (PBEh-3c)

\begin{tabular}{|c|c|c|}
\hline \multirow[t]{2}{*}{ System } & \multicolumn{2}{|c|}{$E_{\text {bind }}\left(\mathrm{kcal} \cdot \mathrm{mol}^{-1}\right)$} \\
\hline & upper rim & lower rim \\
\hline 7, $[\text { creat } \cdots \text { CalixPyr } \cdots \text { creat }]^{++}$ & -28.0 & -2.1 \\
\hline $\mathbf{8 a},[\text { uric ac. } \cdots \text { CalixPyr } \cdots \text { creat }]^{+}$ & -52.1 & -36.0 \\
\hline 9, $[\text { urea } \cdots \text { CalixPyr } \cdots \text { creat }]^{+}$ & -47.9 & -37.5 \\
\hline 10, [creat.. CalixPyr $\cdots$ uric ac. $]^{+}$ & -57.6 & -14.3 \\
\hline 11a, [uric ac. $\cdots$ CalixPyr $\cdots$ uric ac.] & -49.6 & -16.0 \\
\hline 12a, [urea $\cdots$ CalixPyr $\cdots$ uric ac.] & -44.9 & -15.1 \\
\hline 13, $[\text { creat } \cdots \text { CalixPyr } \cdots \text { urea }]^{+}$ & -59.5 & -16.7 \\
\hline 14a, [uric ac. $\cdots$ CalixPyr $\cdots$ urea] & -50.0 & -10.7 \\
\hline 15, [urea $\cdots$ CalixPyr $\cdots$ urea] & -44.6 & -9.6 \\
\hline 16, $[\text { creat } \cdots \text { CalixPyr } \cdots \mathrm{Na}]^{++}$ & -16.2 & -33.5 \\
\hline 17, $[\text { creat } \cdots \text { CalixPyr } \cdots \mathrm{K}]^{++}$ & -18.9 & -22.2 \\
\hline 18, $\left[\text { creat } \cdots \text { CalixPyr } \cdots \mathrm{NH}_{4}\right]^{++}$ & -19.9 & -21.0 \\
\hline
\end{tabular}

obtained by Jaffé method. 20]

It was observed that sample filtration did not improve the results obtained; therefore, that step was omitted (see Figure S4), making it easier to use the $\mu \mathrm{TAD}$. Additionally, a test dilution study with one real urine sample demonstrated that the optimal dilution was 1:100, obtaining optimal way creatinine values and avoiding the interference that affects the $\mu$ TAD (see Table S5). Finally, the kinetic study confirmed that $30 \mathrm{~s}$ was the optimal measurement time for urine samples. From $45 \mathrm{~s}$ onwards, the membrane produced an orange colour that was not due to the creatinine present. It was most likely due to the generation of ammonia from the urea, which changed the $\mathrm{pH}$ and turned the indicator to lower $H$ values, including values beyond the range of the Boltzmann equation.

Once the sample treatment was optimized, seven urines were analysed using the $\mu \mathrm{TAD}$ and the values obtained with a reference method compared. The results obtained are presented in Table 5 . 

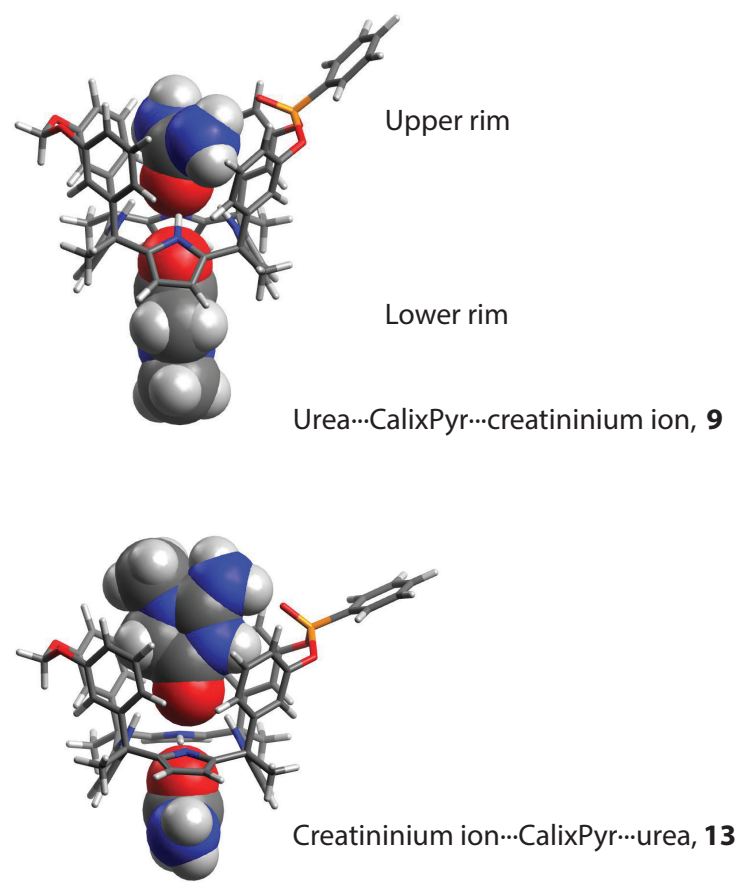

Figure 7: Optimized structures of $[\text { urea } \cdots \text { CalixPyr } \cdots \text { creat }]^{+} \quad(\mathbf{9})$ and $[\text { creat... CalixPy } \cdots \text { urea }]^{+}(\mathbf{1 3})$, optimized at the DFT theoretical level (PBEh-3c).

\section{Conclusions}

In this work, we developed a colorimetric $\mu \mathrm{TAD}$ based on commercial cotton thread where all the reagents are immobilized to determine creatinine in urine based on an ionophore-chromoionophore chemistry.

The ionophore was included in the device using cotton as a support, making a fast determination of creatinine $(30 \mathrm{~s})$ possible, using a very low volume of sample $(10 \mu \mathrm{L})$ and reagents $(0.5 \mu \mathrm{L})$. As a result, it is very cheap $(0.02 € /$ unit $)$ and environmentally friendly due to the low residues generated after use and the use of a smartphone to detect the sensing area and obtain the creatinine concentration. The combination of microfluidic devices based on thread, together with smartphones running an app (recognizing ROI and calculating its $H$ parameter), allows patients and users to monitor the analytes by themselves in an easy and fast way, bringing the laboratory to the sample. Thus, future work 
Table 5: Determination of creatinine for urine samples using Jaffé as the reference method

\begin{tabular}{cccc}
\hline Urine sample & $\mu$ TAD $\mathrm{mM}$ & Jaffé $\mathrm{mM}$ & $\mid$ Error $\mid \%$ \\
\hline S-1 & 11.8 & 10.4 & 13.6 \\
S-2 & 3.8 & 4.6 & 16.7 \\
S-3 & 3.1 & 3.2 & 4.7 \\
S-4 & 18.1 & 17.4 & 3.9 \\
S-5 & 27.4 & 22.9 & 19.7 \\
S-6 & 7.6 & 9.0 & 15.7 \\
S-7 & 21.3 & 17.9 & 19.0 \\
\hline
\end{tabular}

must focus on simplifying and automating the analysis procedure by including analytical operations like filtration or the dilution of the sample in the $\mu \mathrm{TAD}$.

The CalixPyr used as ionophore was studied in depth using density functional (DFT) calculations to characterize the interaction between the ionophore and creatininium ion. The computational results indicate a synergistic mechanism with other chemical species (urea, uric acid and other cations) between the two binding sites of the ionophore, which enhances the creatininium $\cdots$ ionophore binding energies. The $E_{b i n d}$ values of CalixPyr and alkaline ions such as $\mathrm{Na}^{+}$, $\mathrm{K}^{+}$and $\mathrm{NH}_{4}^{+}$were larger for the lower rim of the CalixPyr moiety, whereas for the creatininium cation, urea and uric acid, the largest $E_{b i n d}$ values corresponded to the upper rim. However, the ternary complexes [creatininium ion $\cdots$ Calixpyr $\cdots$ urea $]^{+}$complexes $\mathbf{9}$ and $\mathbf{1 3}$ had the largest $E_{\text {bind }}$ values.

\section{Acknowledgement}

This work was founded by Spanish "Ministerio de Economía y Competitividad" under Project CTQ2016-78754-C2-1-R. Financial support from Junta de Andalucía (FQM-174) is acknowledged. We also thank the "Centro de Servicios de Informática y Redes de Comunicaciones" (CSIRC) (Alhambra Supercomputer Center), University of Granada, for providing computing time. 


\section{References}

[1] J. Delanghe, C. Cobbaert, M.-M. Galteau, A. Harmoinen, R. Jansen,

365

[5] G. Mistlberger, G. Crespo, E. Bakker, Ionophore-based optical sen口 annurev-anchem-071213-020307.

[6] M. M. Erenas, I. de Orbe-Payá, L. F. Capitan-Vallvey, Surface modified 385

口 thread-based microfluidic analytical device for selective potassium analysis, Anal. Chem. 88 (10) (2016) 5331-5337. doi:10.1021/acs.analchem. $6 \mathrm{~b} 00633$

[7] I. H. A. Badr, R. D. Johnson, M. J. Madou, L. G. Bachas, Fluorescent ion-selective optode membranes incorporated onto a centrifugal microflu- 
idics platform, Anal. Chem. 74 (21) (2002) 5569-5575. doi:10.1021/ ac025690z,

[8] R. D. Johnson, I. H. A. Badr, G. Barrett, S. Lai, Y. Lu, M. J. Madou, L. G. Bachas, Development of a fully integrated analysis system for ions based on ion-selective optodes and centrifugal microfluidics, Anal. Chem. 73 (16) (2001) 3940-3946. doi:10.1021/ac0102819

[9] H. Hisamoto, T. Horiuchi, M. Tokeshi, A. Hibara, T. Kitamori, On-chip integration of neutral ionophore-based ion pair extraction reaction, Anal. Chem. 73 (6) (2001) 1382-1386. doi:10.1021/ac001271v.

[10] N. Nuriman, B. Kuswandi, W. Verboom, Optical fiber chemical sensing of hg(ii) ions in aqueous samples using a microfluidic device containing a selective tripodal chromoionophore-pvc film, Sens. Actuators, B 157 (2011) 438-443. doi:10.1016/j.snb.2011.04.084.

[11] X. Wang, M. Sun, S. A. Ferguson, J. D. Hoff, Y. Qin, R. C. Bailey, M. E. Meyerhoff, Ionophore-based biphasic chemical sensing in droplet microfluidics, Angew. Chem. Int. Ed. 58 (24) (2019) 8092-8096.

[12] C. Gerold, E. Bakker, C. Henry, Selective distance-based k+ quantification on paper-based microfluidics, Anal. Chem. 90 (2018) 4894-4900.

[13] T. Guinovart, D. Hernández-Alonso, L. Adriaenssens, P. Blondeau, F. X. Rius, P. Ballester, F. J. Andrade, Characterization of a new ionophorebased ion-selective electrode for the potentiometric determination of creatinine in urine, Biosens. Bioelectron. 87 (2017) 587-592. doi:10.1016/j. bios.2016.08.025,

[14] M. M. Erenas, I. Ortiz-Gómez, I. de Orbe-Payá, D. Hernández-Alonso, P. Ballester, P. Blondeau, F. J. Andrade, A. Salinas-Castillo, L. F. CapitánVallvey, Ionophore-based optical sensor for urine creatinine determination, ACS Sens. 4 (2) (2019) 421-426. doi:10.1021/acssensors .8b01378. 
[15] Á. Sánchez-González, F. J. Martín-Martínez, J. A. Dobado, The role of weak interactions in lignin polymerization, J. Mol. Model. 23 (3) (2017) 80. doi:10.1007/s00894-017-3257-4.

[16] I. Alkorta, F. Blanco, J. Elguero, J. Dobado, S. M. Ferrer, I. Vidal, Carbon $\cdots$ carbon weak interactions, J. Phys. Chem. A 113 (29) (2009) 8387-8393. doi:10.1021/jp903016e

[17] N. M. Settergren, P. Bühlmann, E. A. Amin, Assessment of density functionals, semiempirical methods, and scc-dftb for protonated creatinine geometries, Theochem 861 (1-3) (2008) 68-73. doi:10.1016/j.theochem. 2008.04 .010

[18] J. Gao, Y. Hu, S. Li, Y. Zhang, X. Chen, Tautomeric equilibrium of creatinine and creatininium cation in aqueous solutions explored by raman spectroscopy and density functional theory calculations, Chem. Phys. 410 (2013) 81-89. doi:10.1016/j.chemphys.2012.11.002.

[19] M. M. Erenas, B. Carrillo-Aguilera, K. Cantrell, S. Gonzalez-Chocano, I. M. Perez de Vargas-Sansalvador, I. de Orbe-Payá, L. F. Capitan-Vallvey, Real time monitoring of glucose in whole blood by smartphone, Biosensors and Bioelectronics 136 (2019) 47-52. doi:10.1016/j.bios.2019.04.024

[20] J. M. Pizzolante, Stabilized alkaline picrate reagent for jaffe creatinine determination, uS Patent 4,818,703 (april 4 1987).

URL https://patents .google.com/patent/US4818703A

[21] F. Neese, The orca program system, Wiley Interdisciplinary Reviews: Computational Molecular Science 2 (2012) 73-78. doi:10.1002/wcms.81

[22] F. Neese, Software update: the orca program system, version 4.0, Wiley Interdisciplinary Reviews: Computational Molecular Science 8 (1) (2017) e1327. doi:10.1002/wcms.1327.

[23] S. Grimme, J. G. Brandenburg, C. Bannwarth, A. Hansen, Consistent structures and interactions by density functional theory with small atomic 
orbital basis sets, J. Chem. Phys. 143 (5) (2015) 054107. doi:10.1063/1. 4927476 .

[24] M. C. Daza, J. Dobado, J. Molina, P. Salvador, M. Duran, J. Villaveces, Basis set superposition error-counterpoise corrected potential energy surfaces. Application to hydrogen peroxide $\cdots \mathrm{X}$ (X=F-, Cl-, Br-, $\mathrm{Li}+$, $\mathrm{Na}+$ ) complexes, J. Chem. Phys. 110 (24) (1999) 11806-11813. doi: 10.1063/1.479166.

[25] H. Kruse, S. Grimme, A geometrical correction for the inter- and intramolecular basis set superposition error in hartree-fock and density functional theory calculations for large systems, J. Chem. Phys. 136 (15) (2012) 154101. doi:10.1063/1.3700154.

[26] S. Grimme, J. Antony, S. Ehrlich, H. Krieg, A consistent and accurate ab initio parametrization of density functional dispersion correction (dft-d)

a for the 94 elements h-pu, J. Chem. Phys. 132 (15) (2010) 154104. doi: $10.1063 / 1.3382344$

[27] G. Stefan, E. Stephan, G. Lars, Effect of the damping function in dispersion corrected density functional theory, J. Comput. Chem. 32 (7) (2011) 14561465. doi:10.1002/jcc.21759.

[28] E. Bakker, P. Bühlmann, E. Pretsch, Carrier-based ion-selective electrodes and bulk optodes. 1. general characteristics, Chem. Rev. 97 (8) (1997) 3083-3132. doi:10.1021/cr940394a

[29] K. Cantrell, M. M. Erenas, I. de Orbe-Payá, L. F. Capitán-Vallvey, Use of the hue parameter of the hue, saturation, value color space as a quantitative analytical parameter for bitonal optical sensors, Anal. Chem. 82 (2) (2010) 531-542. doi:10.1021/ac901753c.

470 [30] M. M. Erenas, K. Cantrell, J. Ballesta-Claver, I. de Orbe-Payá, L. F. Capitán-Vallvey, Use of digital reflection devices for measurement using 
[31] Y. Mi, E. Bakker, Lipophilic ionic sites for solvent polymeric membrane ph

[32] Y. Mi, C. Green, E. Bakker, Polymeric membrane ph electrodes based on electrically charged ionophores, Anal. Chem. 70 (24) (1998) 5252-5258. doi:10.1021/ac9806781.

[33] A. Nilghaz, D. R. Ballerini, W. Shen, Exploration of microfluidic devices based on multi-filament threads and textiles: A review, Biomicrofluidics 7 (5) (2013) 051501. doi:10.1063/1.4820413.

[34] R. Malon, L. Heng, E. Córcoles, Recent developments in microfluidic paper, cloth-, and thread-based electrochemical devices for analytical chemistry, Rev. Anal. Chem. 36 (4) (2017) 1-19. doi:10.1515/revac-2016-0018.

[35] E. Aydindogan, E. Guler Celik, S. Timur, Paper-based analytical methods for smartphone sensing with functional nanoparticles: Bridges from smart surfaces to global health, Anal. Chem. 90 (21) (2018) 12325-12333. doi: 10.1021/acs. analchem.8b03120. 